\title{
Novel and Efficient Computation of Hilbert-Huang Transform on Surfaces
}

\author{
Jianping $\mathrm{Hu}^{\mathrm{a}}$, Xiaochao Wang ${ }^{\mathrm{b}, *}$, Hong Qin ${ }^{\mathrm{c}}$ \\ ${ }^{a}$ College of Sciences, Northeast Dianli University, Jilin 132012, China \\ ${ }^{b}$ State Key Laboratory of Virtual Reality Technology and Systems, Beihang University, Beijing 100191, China \\ ${ }^{c}$ Department of Computer Science, Stony Brook University (SUNY), Stony Brook, NY 11794-4400, USA
}

\begin{abstract}
Hilbert-Huang Transform (HHT) has proven to be extremely powerful for signal processing and analysis in 1D time series, and its generalization to regular tensor-product domains (e.g., 2D and 3D Euclidean space) has also demonstrated its widespread utilities in image processing and analysis. Compared with popular Fourier transform and wavelet transform, the most prominent advantage of Hilbert-Huang Transform (HHT) is that, it is a fully data-driven, adaptive method, especially valuable for handling non-stationary and nonlinear signals. Two key technical elements of Hilbert-Huang transform are: (1) Empirical Mode Decomposition (EMD) and (2) Hilbert spectra computation. HHT's uniqueness results from its capability to reveal both global information (i.e., Intrinsic Mode Functions (IMFs) enabled by EMD) and local information (i.e., the computation of local frequency, amplitude (energy) and phase information enabled by Hilbert spectra computation) from input signals. Despite HHT's rapid advancement in the past decade, its theory and applications on surfaces remain severely under-explored due to the current technical challenge in conducting Hilbert spectra computation on surfaces. To ameliorate, this paper takes a new initiative to compute the Riesz transform on 3D surfaces, a natural generalization of Hilbert transform in higher-dimensional cases, with a goal to make the theoretic breakthrough. The core of our theoretic and computational framework is to fully exploit the relationship between Riesz transform and fractional Laplacian operator, which can enable the computation of Riesz transform on surfaces via eigenvalue decomposition of Laplacian matrix. Moreover, we integrate the techniques of EMD and our newly-proposed Riesz transform on 3D surfaces by monogenic signals to compute Hilbert spectra, which include the space-frequency-energy distribution of signals defined over 3D surfaces and characterize key local feature information (e.g., instantaneous frequency, local amplitude, and local phase). Experiments and applications in spectral geometry processing and prominent feature detection illustrate the effectiveness of the current computational framework of HHT on 3D surfaces, which could serve as a solid foundation for upcoming, more serious applications in graphics and geometry computing fields.
\end{abstract}

Keywords:

EMD, Hilbert spectral analysis, Riesz transform, Fractional Laplacian operator

\section{Introduction and Motivation}

With the rapid development of data acquisition devices and computing resources, diverse types of data are acquired routinely at an accelerated pace everyday. Novel data modeling, processing, and analysis methods must be developed in concert to address new technical challenges, and this is especially urgent when we are facing the big data challenge at present.

Hilbert-Huang transform (HHT), a fully data-adaptive multiscale data analysis and processing method, had been proposed initially by Huang et al. [1] in 1998. Ever since its inception, it has gained tremendous popularities, and hence has proven to be extremely powerful for signal processing and analysis in 1D time series. As a result, it has

\footnotetext{
${ }^{*}$ Corresponding author

Email address: wangxiaochao18@163.com (Xiaochao Wang)
} 

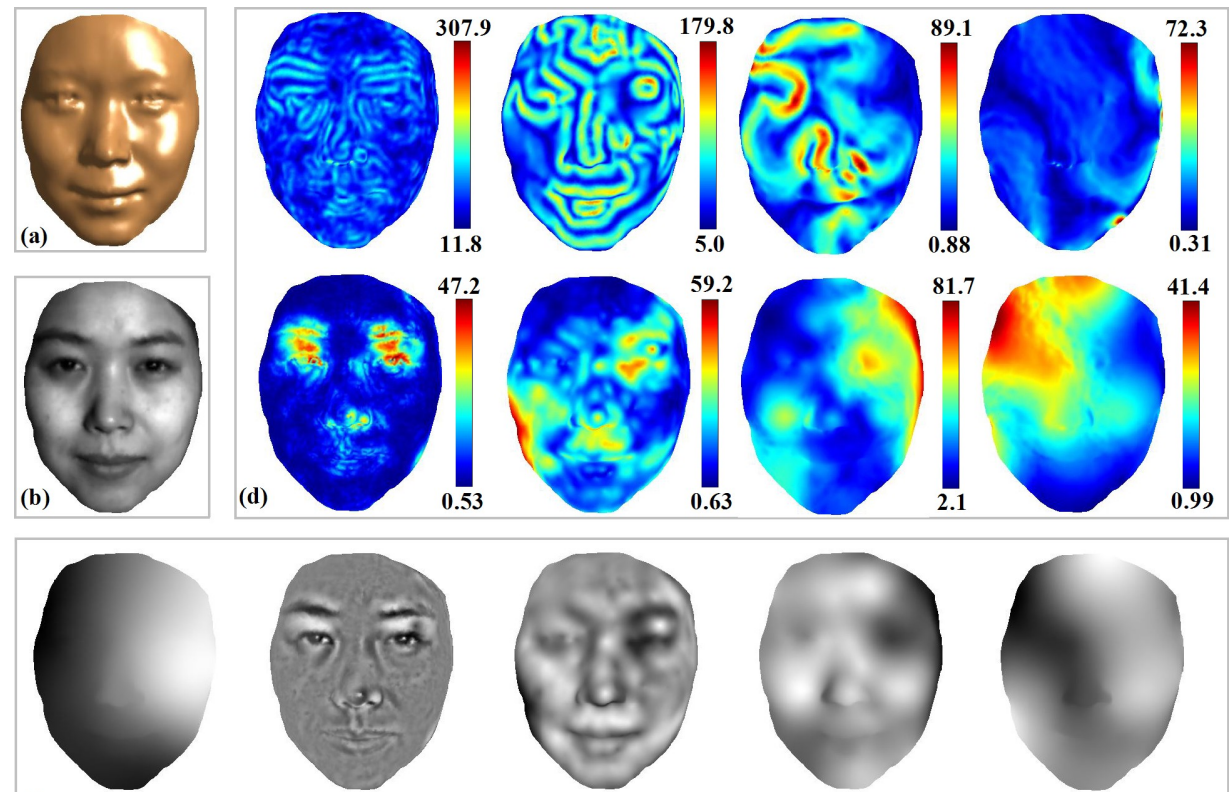

(c) Residue

IMF1

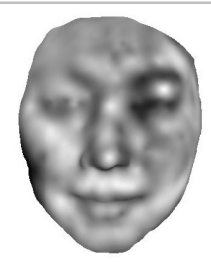

IMF2

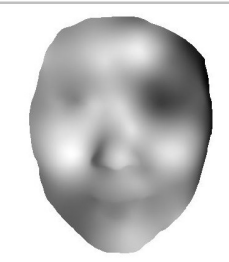

IMF3

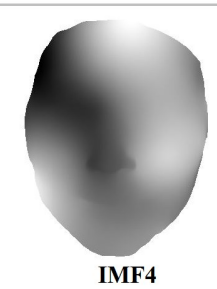

Figure 1: Hilbert-Huang Transform (HHT) for a signal defined on a 3D model. (a) The initial face model. (b) The texture signal. (c) Empirical mode decomposition with four IMFs and a residue. (d) Hilbert spectral analysis. The first row and the second row represent the instantaneous frequency and amplitude of the corresponding IMFs respectively.

yielded a wide variety of applications in many science and engineering fields [2], such as geophysics [3], atmospheric [4], climate [5], oceanographic studies [6], geology [7], and many more. The generalization of HHT to higherdimensional, regular, tensor-product domains (e.g., 2D and 3D Euclidean space) has also demonstrated its widespread utilities in handling higher-dimensional data towards more quantitative processing and more accurate analysis. So far, typical applications include (but not are limited to): image compression [8][9][10], image analysis [11][12], image fusion [13], facial emotion recognition [14], skeleton pruning [15], field simulation and modulation [16][17], etc.

Compared with traditional techniques such as Fourier analysis, short-time Fourier analysis, and wavelet analysis, the most prominent advantage of HHT is that, it is a fully data-driven, adaptive method, especially valuable for processing and analyzing non-stationary and nonlinear signals [1]. Its initial success in handling 1D time series clearly exhibits its potential in data analysis, and its strength is also echoed by its rapid generalization to image domains. The core technical elements of HHT are Empirical Mode Decomposition (EMD) and Hilbert spectral analysis. The goal of EMD is to decompose a signal into a finite number of Intrinsic Mode Functions (IMFs) with multi-scale oscillatory modes from fine to coarse and a residue with monotonic trend [2]. Such IMFs as bases are global, completely data-adaptive and they differ substantially among different signals. It may be noted that, through the use of the sifting process, EMD only offers a set of empirical bases that are totally data-driven and tend to be much fewer than other well-known bases (e.g., Fourier bases, wavelet bases) [1][2]. In order to glean more meaningful local information towards more quantitative analysis from such IMFs after EMD is carried out, we must resort to Hilbert transform [18][19], that essentially computes the time/space-frequency-energy distribution of a signal from each IMF (i.e., Hilbert spectra). Without Hilbert transform, we will be unable to pinpoint local frequency, energy, and phase information precisely. In technical essence, HHT's unique strength towards more quantitative and more accurate analysis results from its superior capability to reveal both global information (i.e., Intrinsic Mode Functions (IMFs) enabled by EMD) and local information (i.e., the computation of instantaneous frequency, phase, and energy information enabled by Hilbert spectral analysis) from any input signal [1][2].

Despite HHT's rapid development in processing time series and images in 2D/3D domain in the past decade, its theoretic advancement and applications on surface signals are hampered by our inability of conducting Hilbert spectra computation on surfaces. In general, HHT on surface signals remain severely under-explored so far [20]. The primary reason lies in the fact that $3 \mathrm{D}$ surfaces are irregular, curved, and possibly have arbitrarily complex structure which 
will bring forth much more challenges for the computation of the Hilbert transform on surfaces [19]. As a result, prior graphics-related efforts of HHT on surfaces mainly concentrate on identifying multiple intrinsic scales of the input signal via EMD in a global sense [21][22][23] or perform HHT computation via dimensionality reduction[24], and without an accurate and effective way to extract local information from each IMF, we are primarily confined to geometry signal processing such as denoising, smoothing, and enhancement.

Key contributions of this paper are as follows. First, its overarching contribution is primarily at the theoretic front. Specifically, we tackle the challenge of computing the Riesz transform on 3D surfaces, a natural generalization of Hilbert transform in higher-dimensional cases, with an ambitious goal of realizing the full potential of HilbertHuang Transform (HHT) in graphics and visual computing fields. Our computational solution is to take the full advantage of the relationship between Riesz transform and fractional Laplacian operator, which can facilitate the computation of Riesz transform on surfaces via eigenvalue decomposition of Laplacian matrix. Furthermore, we integrate our newly-developed theory and computational techniques in this paper with the feature-sensitive EMD for surface signals [21][23] to compute a Hilbert spectrum that contains the space-frequency-energy distribution of the signals at every location across the entire 3D surface domain (Fig. 1). This paper showcases our initial research endeavor in making HHT on surfaces computationally feasible. Second, at the application front, we explore a small subset of graphics applications to illustrate the effectiveness of the novel HHT theory on 3D surfaces. Based on our extensive experiments on popular geometric models, we are hoping to demonstrate that the Hilbert-Huang transform is invaluable for 3D surface modeling, processing, and analysis. In this paper, our theoretic undertakings would move us one step closer to our ultimate goal, which is to continue to broaden the horizon of HHT's powerful applications in relevant visual computing areas.

\section{Related Work}

We shall briefly review related works of classical time-frequency analysis on 3D surfaces, and HHT (HilbertHuang transform) on regular tensor-product domains and 3D surfaces.

\subsection{Classical Time-frequency Analysis on 3D Surfaces}

Fourier transform is a classical signal analysis tool and could be directly adapted to surfaces, called Fourier transform on surfaces. It can represent shape as superposition of Laplace-Beltrami eigenfunctions (LBEs) analogous to sines and cosines of the Fourier transform in the Euclidean space. This property is first employed by Taubin [25] to design and analyze approximation of low-pass filters. Vallet et al. [26] computed the Fourier transform of signal on the mesh directly. Spherical harmonics (which are the Fourier series on the unit sphere) are used for surface filtering, surface reconstruction, and texture transfer [27][28]. The short-time Fourier transforms are used for processing of point clouds [29]. A more comprehensive survey about its applications in geometry analysis and processing can be found in [30].

Compared with Fourier transform, wavelet transform is more powerful for signal processing because of its locality in both space/time and frequency domain. Subdivision wavelets [31][32] are a natural construction of wavelets on surfaces via explicit subdivision. Despite their earlier success, they are constructed on regular geometric domain via explicit parameterization [32], or curved surfaces satisfying subdivision connectivity [31] which limit their application scope to mesh compression and level-of-detail rendering. Diffusion wavelets are first proposed by Coifman and Maggioni [33] that use a diffusion operator and its dyadic powers for dilation, which generalize the classical wavelets by allowing multiscale analysis on graphs. Hammond et al. [34] proposed spectral graph wavelets being represented by a generating kernel and eigenfunctions of the Laplace operator. Diffusion wavelets and spectral graph wavelets do not need explicit parameterization and subdivision and will result in more extensive applications, such as saliency visualization [35], feature description and detection [36][37][38], shape retrieval [39], mesh compression[40], etc.

\subsection{HHT on Regular Tensor-product Domains}

In 1998, Huang et al. [1] proposed a fully adaptive data analysis and processing method in 1D for time series, called Hilbert-Huang transform, which includes EMD and Hilbert spectral analysis. EMD generates IMFs by a sifting process, which needs to find local extrema points (maxima and minima) and subsequent interpolation of those points 
in each iteration of the process by cubic splines to generate upper and lower envelopes [1]. The time-frequencyenergy distribution of the signal from each IMF can be computed by Hilbert transform. This method can overcome the limitation of Fourier analysis, short-time Fourier analysis, and wavelet analysis that essentially depend on the predefined basis functions and has been applied to various scientific research and engineering fields [3][7][5][6][1][4].

This technique has also been extended to 2D data/images. Linderhed [9] used a thin-plate spline for surface interpolation to develop 2D EMD algorithm for an image compression scheme. Nunes et al. [11] generated envelope surfaces by radial basis function interpolation and used the Riesz transform in 2D Euclidean space(a high dimensional generalization of Hilbert transform) for image analysis. Xu et al. [41] directly used a mesh fitting method based on bicubic spline to compute the local mean surface of the data, which can accelerate the sifting process. Xu et al. [12] used 2D quaternion Hilbert transform to generate a space-frequency-energy distribution of 2D image data and can be applied to texture analysis. Benefited from the powerful ability in data analysis and processing of HHT, other various applications in image domains have also been proposed, such as image fusion [13], facial emotion recognition [14], skeleton pruning [15], etc.

As for signals on more high-dimensional regular tensor-product domains, Pabel et al. [42] extended the 1D EMD method to any dimensional signals with an adaptive wavelet data fitting procedure, and then used Riesz transformation in high-dimensional Euclidean space to derive instantaneous frequency and energy distribution. Gao et al. [16] first used space-filling curves to flatten 3D fluid data into 1D, and then decomposed three 1D signals by the classical 1D EMD method to conduct view-dependent multiscale fluid simulation. Based on the EMD on 3D fluid data, Ren et al. [17] computed spatial Hilbert spectra by 1D Hilbert transform, which can be used flow field modulation.

\subsection{HHT on $3 D$ Surfaces}

Unlike regular tensor-product domains, 3D surfaces are irregular, curved, and possibly have any complex structure, which will unavoidably give rise to more technical challenges when our goal is to extend the coverage of 1D HHT for handling signals defined over surfaces. The earlier method [22] first transforms coordinate functions of 3D surfaces to 2D planar signals with the help of spherical parameterization, and then obtains the EMD representations by the exiting 2D EMD methods. Wang et al. [23] directly generalized 1D EMD to 3D surfaces, which computes the upper and lower envelopes of a function by computing a biharmonic field with Dirichlet boundary conditions. These methods [22][23] directly apply EMD to coordinate functions to conduct 3D surface filtering. Hu et al. [21] developed a novel framework based on EMD using a measure of mean curvature as an input signal, which can help extract features of different scales of the original surface more precisely. Wang et al. proposed an EMD and Hilbert spectra computational scheme to transform the problem of 3D surface analysis/processing to 1D time series processing by the strategy of dimensionality reduction via space-filling curve [24], enabling us to decompose the original signal into IMFs and use the 1D Hilbert transform of a function. However, this method tends to affect the overall accuracy of HHT computation because theoretically it shares common drawbacks of any sampling method in the aspect of information loss.

Despite some limited successes in performing HHT on 3D surfaces in recent years, to the best of our knowledge, none of the aforementioned works directly conduct the Hilbert transform to perform local frequency analysis on 3D surfaces, which has resulted in rather limited applications of HHT being primarily confined to geometry signal processing such as denoising, smoothing, and enhancement. In this paper, we make significantly new efforts to compute the Riesz transform on 3D surfaces, a high dimensional generalization of Hilbert transform, and employ it to compute Hilbert spectra, i.e., a space-frequency-energy distribution of the signal on 3D surfaces, which could in a more quantitative and accurate way characterize critical local feature information (e.g., instantaneous frequency, amplitude, and phase) and will bring forth many more novel applications in graphics and visual computing fields.

\section{Mathematical Elements of HHT}

We start here by first reviewing the mathematical elements of HHT (Hilbert-Huang transform) in 1D [1] briefly, which is comprising two main phases, empirical mode decomposition (EMD) and Hilbert spectral analysis, and then discuss briefly the high dimensional generalization of HHT. 


\subsection{Empirical Mode Decomposition}

The original 1D EMD method [1] extracts a finite number of intrinsic mode functions (IMFs) and a residue from a 1D time series signal. IMFs are band-limited and with local zero mean, which can represent the intrinsic oscillatory modes imbedded in the original data and can be computed iteratively by a sifting process. This sifting process first computes the upper and lower envelopes of signal $f$ using a cubic spline which interpolates all of its local maxima and minima, then averages the upper and lower envelopes to obtain an approximate median $m_{1}$. If $f_{1}=f-m_{1}$ does not satisfy the stopping criteria of sifting process, one can treat $f-m_{1}$ as a new signal and repeat the same process until a satisfactory $f_{1}$ is found and defined as an IMF. After extracting this IMF from the signal, the residue can be used to extract the next IMFs iteratively until the residue is a constant or a monotone trend. Finally, EMD can decompose a given $1 \mathrm{D}$ signal $f$ as

$$
f=\sum_{k=1}^{N} f_{k}+r_{N},
$$

where $f_{k}, k=1, \cdots, N$ are IMFs and $r_{N}$ is the final residue.

\subsection{Hilbert Spectral Analysis}

Once attaining the IMFs generated by EMD, Hilbert spectral analysis [1] can be performed via analytic signal$\mathrm{s}$ [18], which can be generated by Hilbert transform.

As for a 1D function $h(t)$, its Hilbert transform [19] can be defined by

$$
\mathcal{H}(t)=\frac{1}{\pi t} * h(t)=\frac{1}{\pi} \int_{R} \frac{h\left(t^{\prime}\right)}{t-t^{\prime}} d t^{\prime} .
$$

Its corresponding analytic signal $h_{A}(t)$ is formed by a complex conjugate pair consisting of $h(t)$ and $\mathcal{H}(t)$. If the signal $h(t)$ is band-limited and with local zero mean, the analytical signal $h_{A}(t)$ has an orthogonal decomposition into instantaneous amplitude (instantaneous energy) $a(t)$ and instantaneous phase $\phi(t)$, such a property is called a split of identity [43][44], i.e.,

$$
h_{A}(t)=h(t)+\mathbf{i} \mathcal{H}(t)=a(t) e^{\mathbf{i} \phi(t)},
$$

where $\mathbf{i}$ is the imaginary unit, $a(t)$ and $\phi(t)$ can be computed by

$$
a(t)=\sqrt{h(t)^{2}+\mathcal{H}(t)^{2}}, \text { and } \phi(t)=\arctan \frac{\mathcal{H}(t)}{h(t)} .
$$

The instantaneous frequency of the signal $h(t)$ can be defined as the derivative of the instantaneous phase, i.e.,

$$
\omega(t)=\frac{d \phi(t)}{d t} .
$$

When we are applying the Hilbert transform to all IMFs $f_{k}$ of Eq. (1), we are able to get the Hilbert spectra $H(\omega, t)$ of the signal $f$, which can exactly describe the signal amplitude varying on a time-frequency plane. The original signal can be represented by

$$
f(t)=\operatorname{Re}\left(\sum_{k=1}^{N} a_{k}(t) e^{\mathrm{i} \int_{R} \omega_{k}(t) d t}\right)+r_{N}(t),
$$

where $R e$ is the operator of real part, $a_{k}(t)$ and $\omega_{k}(t)$ denote the functions of the instantaneous amplitude and instantaneous frequency of $f_{k}(t)$, respectively.

Compared to the global frequency and energy generated by the Fourier spectral analysis, the instantaneous frequency and energy in Hilbert spectra are very well localized in the time-frequency domain and can reveal important characteristics of the underlying signal. 


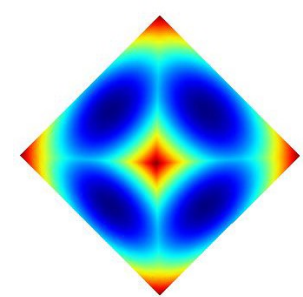

(a)

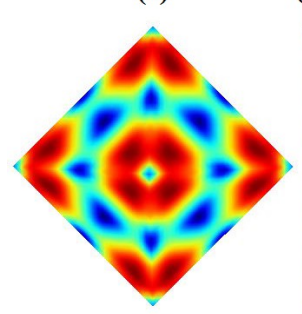

(e)

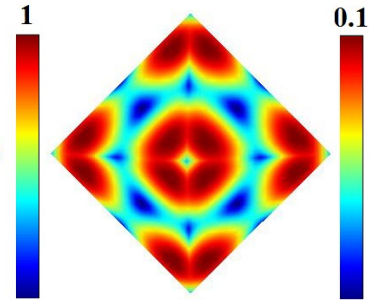

(b)

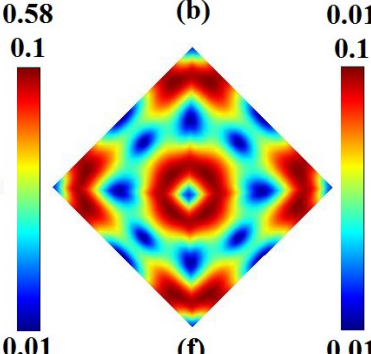

(f)

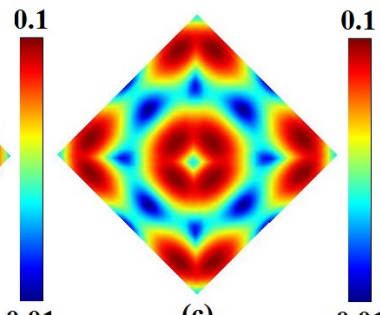

(c)

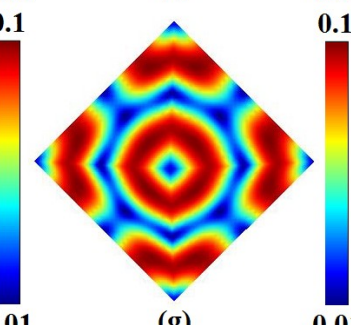

(g)

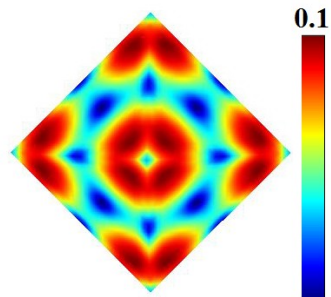

(d)

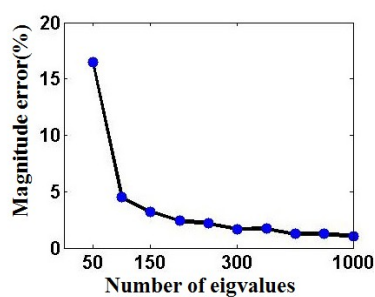

(h)

Figure 2: Illustration the influence of eigenvalue number in the computation of Riesz transform. (a) The central signal computed by the length between the vertex position and the centroid of the Octahedron model. (b) The magnitude of the Riesz transform of (a) generated via full eigenvalue decomposition. The results from (c) to (g) are generated from 1500, 600, 300, 150, and 50 eigenvalues respectively. (h) The magnitude error plots with their deviation from the full eigenvalue decomposition.

\subsection{Generalization of HHT to High Dimensional Domains}

The success of HHT in processing nonlinear and non-stationary signal in 1D has motivated its generalization to 2D image [9][11][12][13], 3D volumetric data [16][17], 3D surface data [21][22][23][24], etc. The extension of EMD to high dimensional domains have been researched extensively where the critical step is the interpolation from extrema at each iteration where various interpolation methods can be used such as radial basis function, thin plate spline, cubic polynomial interpolation and so on. Hilbert spectral analysis in high dimensional domains can also be performed by high dimensional generalization of Hilbert transform including partial Hilbert transform, total Hilbert transform, combined partial and total Hilbert transforms which can also be defined by ways of convolution similar to Eq. (2) [19]. Besides, Riesz transform is another generalization of Hilbert transform and can be defined for a function $h(\mathbf{x})$ in $R^{n}[45][46]$ by

$$
\mathcal{R}_{i} h=\pi \frac{x_{i}}{|\mathbf{x}|^{n+1}} * h(\mathbf{x})=\pi \int_{R^{n}} \frac{\left(x_{i}-x_{i}^{\prime}\right) h\left(\mathbf{x}^{\prime}\right)}{\left|\mathbf{x}-\mathbf{x}^{\prime}\right|^{n+1}} d \mathbf{x}^{\prime}, i=1, \cdots, n .
$$

However, Hilbert transform in high dimensional domains defined by ways of convolution (e.g., Eq. (2) and Eq. (7)) is difficult to compute on 3D surface serving as a domain because 3D surfaces are often curved, and possibly have any complex structure [19]. To the best of our knowledge, the computation method of Hilbert transform on surfaces could not be developed directly in the existing frameworks, which inevitably limits the prior graphics-related efforts in HHT [21][22][23][24].

\section{New Mathematical Formulations of HHT on 3D Surfaces}

Let $M=(\mathbf{V}, \mathbf{K})$ be a triangular mesh which represents a discretization of 3D surface $S$, where $\mathbf{V}$ denotes a set of vertices $\left\{\mathbf{v}_{i}=\left(x_{i}, y_{i}, z_{i}\right) \in R^{3}, i=1, \cdots,|\mathbf{V}|\right\}(|\mathbf{V}|$ denotes the size of the vertex set $\mathbf{V})$, and $\mathbf{K}$ contains all the adjacency information of the mesh including edges $\left\{(i, j) \mid \mathbf{v}_{i}\right.$ and $\mathbf{v}_{j}$ are linked by an edge $\}$ and faces $\left\{(i, j, k) \mid \mathbf{v}_{i}, \mathbf{v}_{j}\right.$ and $\mathbf{v}_{k}$ are the vertices of a triangle $\}$. If the sets of edges and faces are denoted as $\mathbf{E}$ and $\mathbf{F}$ respectively, the triangular mesh $M$ can be represented as $(\mathbf{V}, \mathbf{E}, \mathbf{F})$. Besides, we denote $N(i)=\{j \mid(i, j) \in E\}$ as the vertex set of the 1-ring neighbors of vertex $\mathbf{v}_{i}$.

In the followings, we first describe briefly EMD on 3D surfaces [23], and then introduce our novel computation method about Riesz transform on 3D surfaces and detail the new method on how to obtain Hilbert spectra via monogenic signals. 


\subsection{EMD on $3 D$ Surfaces}

As for a given function $f$ defined on a 3D surface, EMD can iteratively generate the $k$-th IMF $f_{k}$ from the $(k-1)$ th residue $r_{k-1}\left(r_{0}=f\right)$ by a sifting process [23]. The entire decomposition pipeline is similar to the 1D case, where the interpolation method for computing envelopes from local extrema of the sifting process are conducted over 3D surfaces serving as domains.

A biharmonic field with Dirichlet boundary is constructed to generate the upper and lower interpolation envelopes in EMD on surfaces [23]. It is a generalization of the cubic spline to 3D surface and minimizes the thin-plate energy of a function $g$ defined on a 3D surface, and the Euler-Lagrange equation of this energy formulation is

$$
\Delta^{2} g=0,
$$

where $\Delta$ is the Laplace-Beltrami operator on the surface.

The discrete Laplace-Beltrami operator on a meshed surface at a vertex $\mathbf{v}_{i}$ can be computed by weighted average over the neighborhood

$$
\Delta\left(\mathbf{v}_{i}\right)=\sum_{j \in N(i)} w_{i j}\left(\mathbf{v}_{j}-\mathbf{v}_{i}\right)
$$

and can be formulated as a Laplacian matrix with the following elements

$$
\mathbf{L}_{i j}=\left\{\begin{array}{ll}
\sum_{k \in N(i)} w_{i k}, & j=i \\
-w_{i j}, & j \in N(i) \\
0, & \text { otherwise }
\end{array} .\right.
$$

If we denote $\left\{\left(\mathbf{v}_{i}, f\left(\mathbf{v}_{i}\right)\right), i \in C\right\}$ as the given data constraints, the interpolation function $\mathbf{g}=\left(g\left(\mathbf{v}_{1}\right), g\left(\mathbf{v}_{2}\right), \cdots, g\left(\mathbf{v}_{|\mathbf{V}|}\right)\right)$ at each vertex of the mesh $M$ can be computed by solving the following $|\mathbf{V}| \times|\mathbf{V}|$ linear system

$$
\mathbf{L}^{2} \cdot \mathbf{g}=0, \text { s.t., } g\left(\mathbf{v}_{i}\right)=f\left(\mathbf{v}_{i}\right), i \in C,
$$

where $\mathbf{L}$ is the $|\mathbf{V}| \times|\mathbf{V}|$ discretized Laplacian matrix (Eq. (10)) with cotangent weight $w_{i j}=\frac{1}{2 A_{i}}\left(\cot \alpha_{i j}+\cot \beta_{i j}\right.$ ) [47], $\alpha_{i j}$ and $\beta_{i j}$ are the angles opposite to the mesh edge $(i, j)$, and $A_{i}$ is the Voronoi area of vertex $\mathbf{v}_{i}$.

\subsection{Riesz Transform on 3D Surfaces}

Our novel computational scheme about Riesz transform on 3D surfaces is to take the full advantage of the important connection between Riesz transform and the fractional Laplacian operator [45][46], which can be computed via a spectral definition of Laplacian operator.

Specifically, the Riesz transform of a function $h(\mathbf{x})$ in $R^{n}$ defined by Eq. (7) can also be changed into

$$
\mathcal{R}_{i} h=\frac{\partial}{\partial x_{i}} \Delta^{-1 / 2} h, i=1, \cdots, n,
$$

where $\Delta$ is the Laplacian operator with the eigenvalues and eigenfunctions $\left\{\lambda_{k}, \varphi_{k}, k=1, \cdots, n\right\}$, and $\Delta^{-1 / 2}$ is the inverse of the fractional Laplacian operator $\Delta^{1 / 2}$, and can be defined by the spectrum of Laplacian operator [48] as

$$
\Delta^{-1 / 2} h=\sum_{k=1}^{n} \lambda_{k}^{-1 / 2} a_{k} \varphi_{k}, h=\sum_{k=1}^{n} a_{k} \varphi_{k} .
$$

Let $\nabla$ be the gradient operator, the Riesz transform of $h$ defined by Eq. (12) can be written as

$$
\mathcal{R} h=\left(\mathcal{R}_{1} h, \mathcal{R}_{2} h, \cdots, \mathcal{R}_{n} h\right)=\nabla \Delta^{-1 / 2} h .
$$

This Riesz transform defined by Eq. (14) could be extended to a 3D meshed surface $M=(\mathbf{V}, \mathbf{K})$ directly, where the Laplacian operator becomes the Laplace-Beltrami operator and can be discretized by Eq. (9) over the meshed 
surface $M$. According to Eq. (13), we can compute the inverse of the discretized fractional Laplacian operator $\mathbf{L}^{-1 / 2}$ by the eigenvalue decomposition (EVD) of the discretized Laplacian matrix L (Eq. (10)) as follows,

$$
\mathbf{L}^{-1 / 2}=\mathbf{U} \mathbf{R}^{-1 / 2} \mathbf{U}^{\prime}
$$

where $\mathbf{R}=\operatorname{diag}\left(\lambda_{1}, \lambda_{2}, \cdots, \lambda_{|\mathbf{V}|}\right)$ is a diagonal matrix with the eigenvalues of $\mathbf{L}, \mathbf{U}=\left(\varphi_{1}, \varphi_{2}, \cdots, \varphi_{|\mathbf{V}|}\right)$ is a matrix with their corresponding eigenvectors, and $\mathbf{U}^{\prime}$ is the transposition of $\mathbf{U}$.

Combined with Eq.(14) and Eq.(15), the Riesz transform of a signal $h$ defined on the 3D meshed surface $M$ could be generated by computing the gradient of the function $\mathbf{L}^{-1 / 2} h$, i.e.,

$$
\mathcal{R} h=\nabla \mathbf{L}^{-1 / 2} h=\nabla\left(\mathbf{U} \mathbf{R}^{-1 / 2} \mathbf{U}^{\prime} h\right) .
$$

The gradient $\nabla g\left(\mathbf{v}_{i}\right)$ of a scalar function $g$ at each vertex $\mathbf{v}_{i}$, defined as a vector in its local tangent plane, is usually estimated by solving an optimization problem using the finite element method (FEM) [49], i.e.,

$$
\arg \min _{\nabla g\left(\mathbf{v}_{i}\right)} \sum_{j \in N(i)}\left\|\left(\nabla g\left(\mathbf{v}_{i}\right), P_{i j}\right)-\frac{g\left(\mathbf{v}_{i}\right)-g\left(\mathbf{v}_{j}\right)}{\left|\mathbf{v}_{i}-\mathbf{v}_{j}\right|}\right\|^{2},
$$

where $(\cdot, \cdot)$ denotes the inner-product operator, and $P_{i j}$ is the projected unit vector of $\overrightarrow{\mathbf{v}_{i} \mathbf{v}_{j}}$ in the local tangent plane of $\mathbf{v}_{i}$.

The computation of Riesz transform on surfaces in Eq. (16) depends on full eigenvalue decomposition of Laplacian matrix. The time complexity is exponentially growing with respect to the data size, and it is extremely expensive for large datasets in principle. To accelerate the computation, we use partial eigenvalue decomposition to replace full eigenvalue decomposition which is a commonly-used strategy in spectrum geometry processing [30]. Now the formula Eq. (16) becomes

$$
\mathcal{R} h=\nabla \mathbf{L}^{-1 / 2} h \approx \nabla\left(\mathbf{U}_{p} \mathbf{R}_{p}^{-1 / 2} \mathbf{U}_{p}^{\prime} h\right),
$$

where $\mathbf{R}=\operatorname{diag}\left(\lambda_{1}, \lambda_{2}, \cdots, \lambda_{p}\right)$ is a diagonal matrix with the $p$ smallest eigenvalues of $\mathbf{L}, \mathbf{U}_{p}=\left(\varphi_{1}, \varphi_{2}, \cdots, \varphi_{p}\right)$ is a matrix with their corresponding eigenvectors, and $\mathbf{U}_{p}^{\prime}$ is the transposition of $\mathbf{U}_{p}$.

The entire computational pipeline of Riesz transform on 3D surfaces could be illustrated using Algorithm 1. Fig. 2 shows a sequence of the Riesz transform computed with decreasing number of eigenvalues where the input signal is computed by the length between the vertex position and the model centroid. The plots in Fig. 2(h) show the average error between the magnitude of the Riesz transform generated by Eq. (18) and the result computed by Eq. (16), which shows that few hundred smallest eigenvalues suffice to compute the transform faithfully (with small error). In our experiments, we usually compute the lowest 300 eigenvalues which can obtain satisfied results.

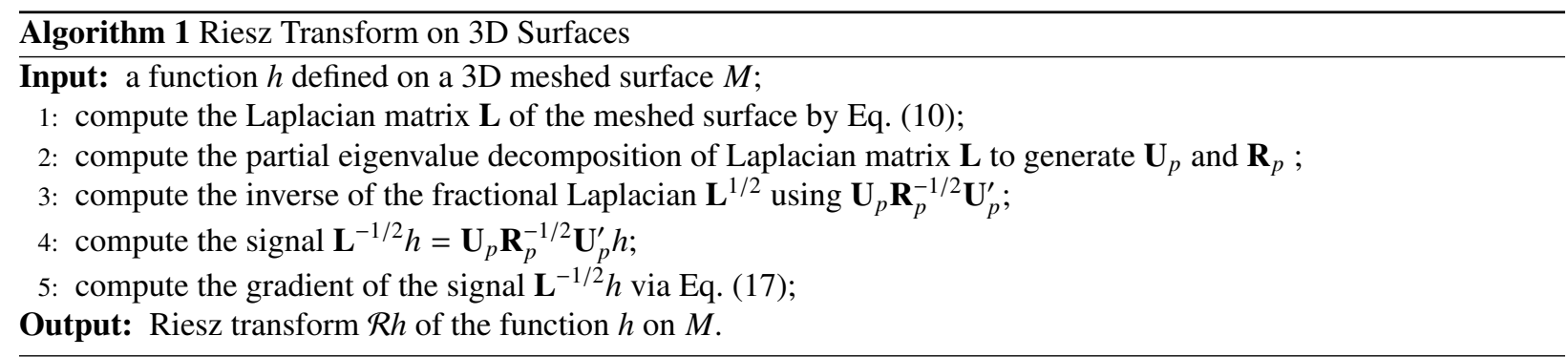

\subsection{Hilbert Spectra Computation on 3D Surfaces based on Monogenic Signals}

The Hilbert spectra of a signal defined on a 3D surface can be computed by monogenic signal [44], which is a high dimensional generalization of analytical signal.

The monogenic signal in 2D Euclidean space is defined by the combination of a 2D signal $h(\mathbf{x}), \mathbf{x} \in R^{2}$ and its Riesz transform $\mathcal{R} h$,i.e.,

$$
h_{R^{2}}(\mathbf{x})=h(\mathbf{x})+(\mathbf{i}, \mathbf{j})\left(\mathcal{R}_{1} h(\mathbf{x}), \mathcal{R}_{2} h(\mathbf{x})\right)
$$




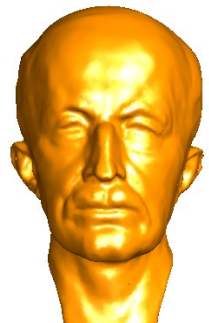

(a)

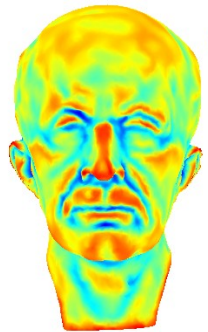

(b)

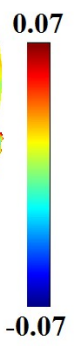

$-\mathbf{0 . 0 7}$

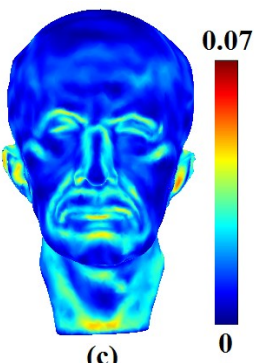

(c)

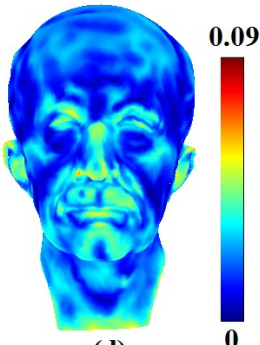

(d)

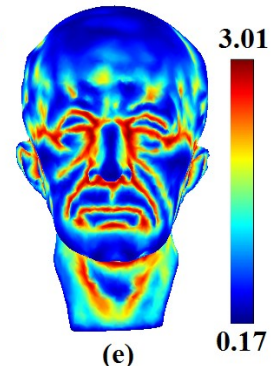

(e)

Figure 3: Local feature-sensitive information extraction and its computation by monogenic signal. (a) The original Maxplanck model. (b) The first IMF of the mean curvature generated by EMD on 3D surfaces. (c) The Riesz transform of (b). (d) and (e) represent the local amplitude and local phase of (c) respectively, where the phase is translated into $[0, \pi]$.

where $\mathbf{i}, \mathbf{j}$ are imaginary units and $\mathcal{R} h(\mathbf{x})=\left(\mathcal{R}_{1} h(\mathbf{x}), \mathcal{R}_{2} h(\mathbf{x})\right)$.

If the signal $h(\mathbf{x})$ is band-limited and with local zero mean, the monogenic signal $h_{R^{2}}(\mathbf{x})$ inherits the property of the analytical signal about the split of identity [43][44]. This property can guarantee $h_{R^{2}}(\mathbf{x})$ is decomposed orthogonally into local amplitude $a(\mathbf{x})$ and local phase $\phi(\mathbf{x})$ which represent the local energetic information and local structural components of the signal and can be computed by $a(\mathbf{x})=\sqrt{h(\mathbf{x})^{2}+\mathcal{R}_{1} h(\mathbf{x})^{2}+\mathcal{R}_{2} h(\mathbf{x})^{2}}$ and $\phi(\mathbf{x})=\arctan \left(\sqrt{\mathcal{R}_{1} h(\mathbf{x})^{2}+\mathcal{R}_{2} h(\mathbf{x})^{2}} / f(\mathbf{x})\right)$ respectively [43]

Naturally, we can generalize the above definition of monogenic signal in 2D by Eq.(19) to 3D surfaces, and it is denoted as

$$
h_{M}(\mathbf{v})=h(\mathbf{v})+(\mathbf{i}, \mathbf{j}, \mathbf{k}) \mathcal{R} h(\mathbf{v}),
$$

where $\mathbf{i}, \mathbf{j}, \mathbf{k}$ are imaginary units, and $\mathcal{R} h(\mathbf{v})=\left(\mathcal{R}_{1} h(\mathbf{v}), \mathcal{R}_{2} h(\mathbf{v}), \mathcal{R}_{3} h(\mathbf{v})\right)$ are the Riesz transform of the signal $h(\mathbf{v})$ defined at each vertex $\mathbf{v}$ of a given 3D meshed surface $M$.

Accordingly, the local amplitude $a(\mathbf{v})$ and local phase $\phi(\mathbf{v})$ of the monogenic signal $h_{M}(\mathbf{v})$ can be computed by

$$
\begin{gathered}
a(\mathbf{v})=\sqrt{h(\mathbf{v})^{2}+\mathcal{R}_{1} h(\mathbf{v})^{2}+\mathcal{R}_{2} h(\mathbf{v})^{2}+\mathcal{R}_{3} h(\mathbf{v})^{2}}, \\
\phi(\mathbf{v})=\arctan \left(\sqrt{\mathcal{R}_{1} f(\mathbf{v})^{2}+\mathcal{R}_{2} f(\mathbf{v})^{2}+\mathcal{R}_{3} f(\mathbf{v})^{2}} / f(\mathbf{v})\right) .
\end{gathered}
$$

They can offer the local feature-sensitive information in image space (i.e., local energy and structure of the image data, respectively) [43], which is inherited naturally in 3D surface case (See Fig. 3)).

Strongly motivated by the important role of the instantaneous frequency (defined as the derivative of the local phase) serving for signal analysis, we propose to exploit the magnitude of the gradient of the local phase as the instantaneous frequency in 3D surface case, i.e.,

$$
\omega(\mathbf{v})=\|\nabla \phi(\mathbf{v})\|_{2} .
$$

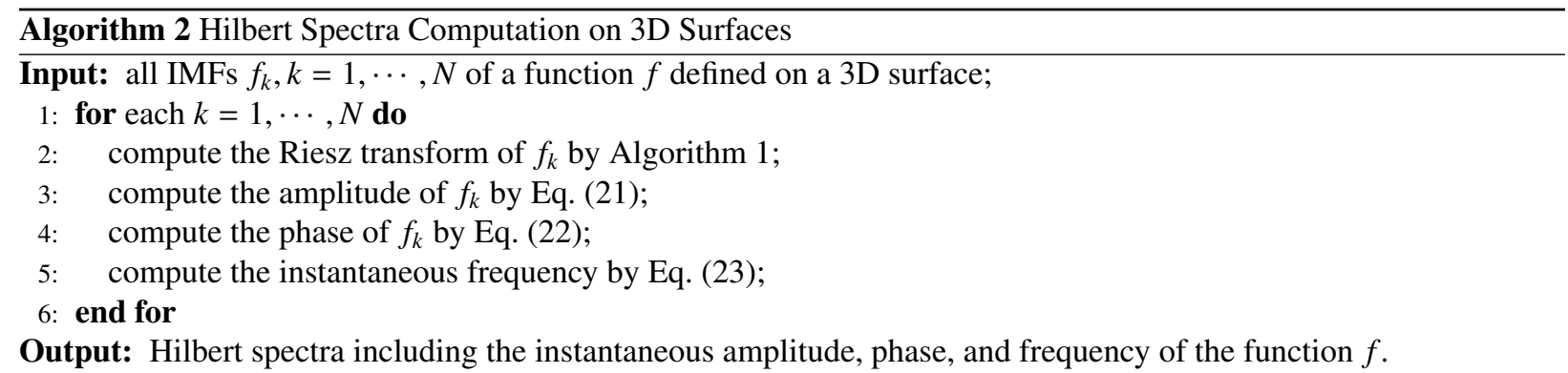

When we are applying the Riesz transform to all IMFs $f_{k}, k=1, \cdots, N$ of the signal $f$ to compute the instantaneous frequency and amplitude, we are able to compute the Hilbert spectra $H(\omega, \mathbf{v})$ that essentially comprise the spacefrequency-energy distribution, which can exactly characterize the signal amplitude varying on 3D surface for all 


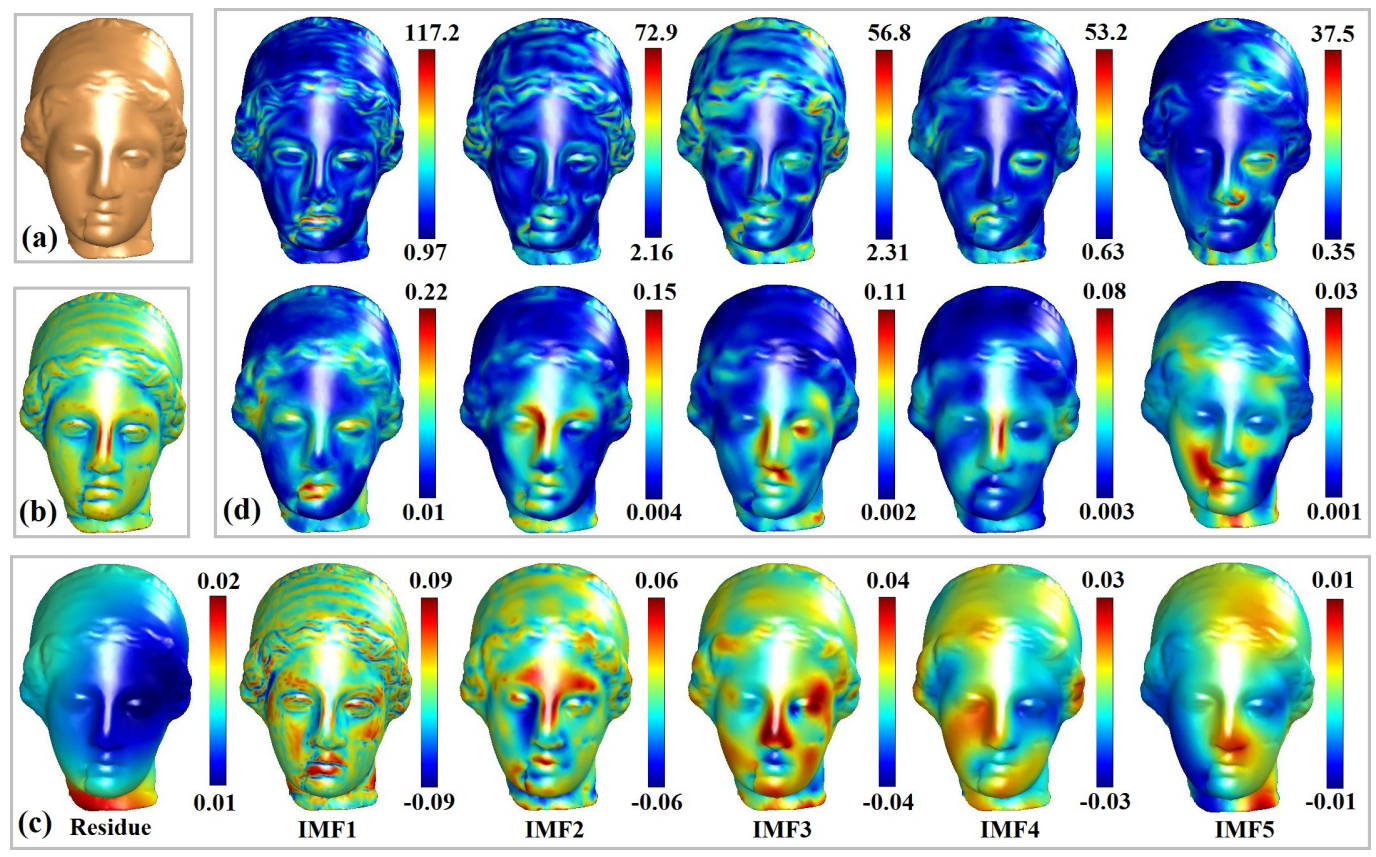

Figure 4: Hilbert-Huang Transform (HHT) for a signal defined on a 3D model. (a) The Hygeia model. (b) The mean curvature serving as a signal. (c) Empirical mode decomposition with five IMFs and a residue. (d) Hilbert spectral analysis. The first row and the second row of (d) represent the instantaneous frequency and amplitude of the corresponding IMF, respectively.

possible signal frequency in a much more quantitative and accurate manner (Fig. 1 and Fig.4). Algorithm 2 describes the entire computational procedure of the Hilbert spectra of a given signal defined on a 3D surface.

\section{Experiment Results and Applications}

We have implemented the above-mentioned algorithms of Hilbert-Huang transform on 3D surfaces in Matlab 2014 on a computer with Inter Core (TM) i7 computer with $16.0 \mathrm{~GB}$ RAM, and rigorously tested them on many models with mesh size from $4 k$ to $100 k$. The main time-consuming parts of our method include the decomposition of the signal by EMD and the computation of the Riesz transform of all IMFs. EMD requires a sifting process in an iterative way to extract each IMF and two sparse linear systems must be solved in each iteration. Riesz transform would need to compute the eigenvalue decomposition of Laplacian matrix which is the most time-consuming part in our algorithm. The timing statistics of some tested models in our experiments are documented in Table 1.

Fig. 1 and Fig. 4 provide the Hilbert-Huang transform results for the texture signal and the mean curvature signal defined on two 3D surfaces, respectively. These two signals are decomposed into several IMFs using EMD which exhibit quantitative features with the different scales of the signals, i.e., the first IMF represents the finest spatial scale, the subsequent IMFs offer much coarser spatial scales in a whole, etc. However, the leading IMFs in Fig. 1 and Fig. 4 have some oscillatory modes even in flat region(e.g. face and neck), therefore it is inaccurate to regard the serial number of IMF as a measure of frequency. This phenomenon means every IMF has a varying instantaneous frequency distribution and has band overlaps in their frequency ranges, which is called mode mixing [17][50]. It is a drawback of the original 1D EMD and is inherited in the 3D surface setting. This side effect can be reduced by applying our novel Riesz transform to all IMFs to generate Hilbert spectra with a space-frequency-energy distribution, which can characterize the signal amplitude variation on 3D surfaces for all possible signal frequency. This distribution demonstrates the frequency values are smaller in flat regions(e.g. face and neck) while they are larger in curved regions such as hair, eye, mouth, etc.

In fact, Hilbert-Huang Transform on 3D surfaces generates a set of adaptive bases by EMD and an instantaneous frequency energy distribution by Riesz transform from the data, and such process has shown its unique advantage 


\begin{tabular}{llllll}
\hline \multirow{2}{*}{ Models } & \multirow{2}{*}{ \#Vertices } & \multirow{2}{*}{ \#IMFs } & & \multicolumn{2}{c}{ Timing(s) } \\
\hline Octahedron(Fig. 2) & & & EMD & RT & Total \\
Hygeia(Fig. 4) & 4,098 & 3 & 0.6 & 5.13 & 6.73 \\
Dragon(Fig. 5(The second row)) & 13,424 & 5 & 3.8 & 24.37 & 31.45 \\
Hand(Fig. 9(c)) & 31,124 & 8 & 10.9 & 110.81 & 137.21 \\
Tyrannosaur(Fig. 9(a)) & 61,563 & 13 & 38.2 & 238.76 & 303.32 \\
\hline
\end{tabular}

Table 1: Run time statistics. EMD: time of performing EMD. RT: time of performing the Riesz transform. Total: total time.

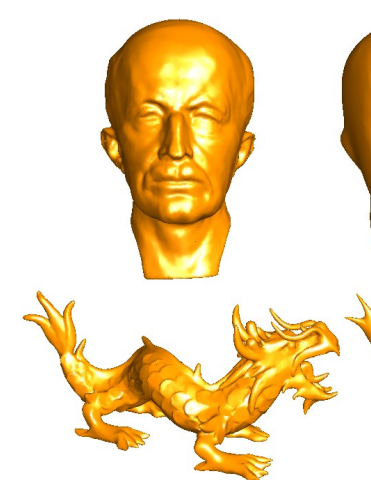

(a)
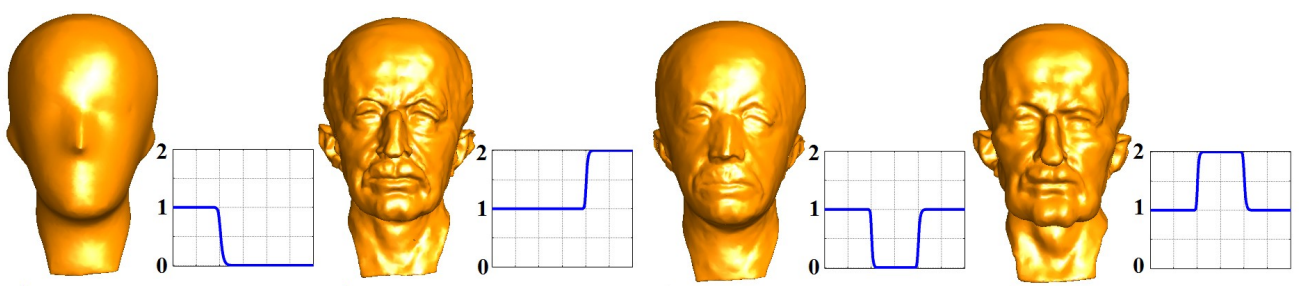

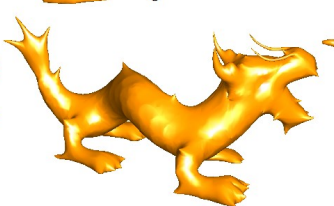

(b)

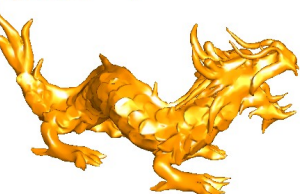

(c)

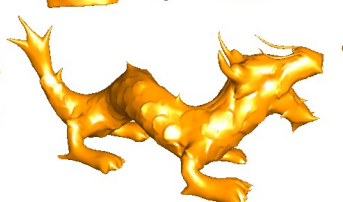

(d)

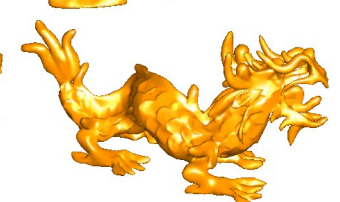

(e)

Figure 5: Various tasks in spectral geometry processing. (a) The original Maxplanck model (The first row) and Dragon model (The second row). (b) Low-pass filtering. (c) High-frequency enhancement filtering. (d) Band-stop filtering. (e) Band-enhancement filtering.

in dealing with non-linear and non-stationary signals in Euclidean spaces. And naturally, we are expecting that such computational framework shall be highly promising in 3D surface analysis and processing, and many geometry analysis and processing tasks can be accommodated, such as spectral geometry processing, feature detection, shape description, saliency visualization, and so on. In the subsequent sections, (in the interest of space) we will focus on the applications of spectral geometry processing and prominent feature detection to illustrate the effectiveness of the novel HHT theory on 3D surfaces.

\subsection{Spectral Geometry Processing}

Hilbert-Huang transform on 3D surfaces enable geometry processing at different frequencies and places, by filtering their amplitude coefficients. In this application, a measure of mean curvature is taken as the input signal, which has more advantages in Hilbert-Huang transform for signal design and processing than the use of coordinate functions as introduced in [21]. It is defined as the inner-product of discretized Laplacian operator Eq. (9) at the vertex $\mathbf{v}_{i}$ and the corresponding vertex normal as follows:

$$
s\left(\mathbf{v}_{i}\right)=\left(\Delta\left(\mathbf{v}_{i}\right) \cdot \mathbf{n}_{i}\right) .
$$

Accordingly, we are able to compute the Hilbert-Huang transform of the signal $s$ in Eq. (24) to obtain Hilbert spectra that essentially comprise the space-frequency energy distributions which can rewrite the input signal $s$ as

$$
s=\operatorname{Re}\left(\sum_{k=1}^{N} a_{k}(\mathbf{v}) e^{\mathbf{i} \int_{M} \omega_{k}(\mathbf{v}) d \mathbf{v}}\right)+r_{N},
$$

where $a_{k}(\mathbf{v})$ and $\omega_{k}(\mathbf{v})$ are the instantaneous amplitude and frequency respectively, and $r_{N}$ is the residue. Then we can design various 3D surface filters $\mu\left(\omega_{k}(\mathbf{v})\right)$ guided by Hilbert spectra to conduct many quantitative and meaningful processing as

$$
s^{\prime}=\operatorname{Re}\left(\sum_{k=1}^{N} a_{k}(\mathbf{x}) \mu\left(\omega_{k}(\mathbf{x})\right) e^{\mathbf{i} \int_{M} \omega_{k}(\mathbf{x}) d \mathbf{x}}\right)+r_{N} .
$$

These filters include low-pass filtering, high-frequency enhancement filtering, band-stop filtering, and band-enhancement filter, etc. 


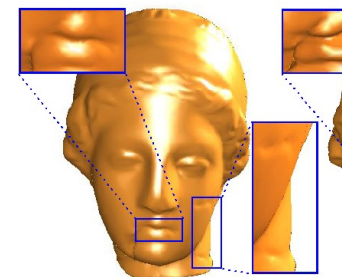

(a)

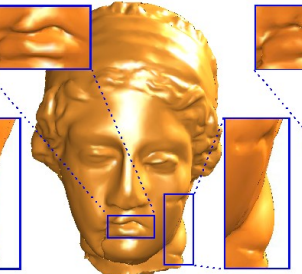

(b)

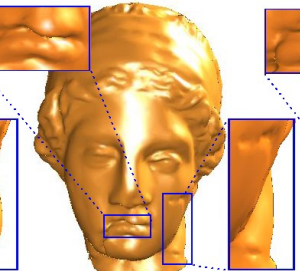

(c)

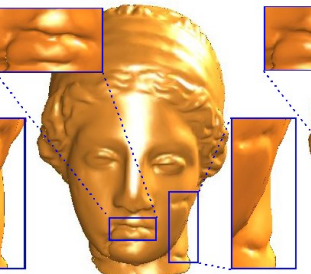

(d)

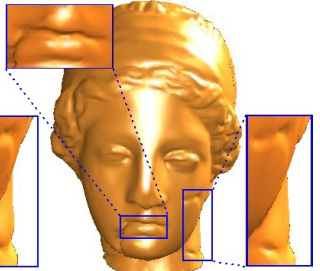

(e)

Figure 6: A comparison of detailed feature enhancement with related methods. (a) The original Hygeia model. (b) The result generated by enhancing the first IMF 2.5 times [21]. (c) to (e) are the results generated by enhancing the amplitude 2.5 times in the frequency range of detailed features by the method in [24], manifold harmonics [26], and our method respectively.

Finally, a linear system can be effectively used to reconstruct a new mesh $\mathbf{V}^{\prime}$ corresponding to the new signal $s^{\prime}$ in the least squares sense with the original vertex positions $\mathbf{V}$ as constraints, and can be described as

$$
\left[\frac{\mathbf{L}}{\mu \mathbf{I}}\right] \mathbf{V}^{\prime}=\left[\frac{\operatorname{diag}\left(\mathbf{s}^{\prime}\right) \mathbf{N}}{\mu \mathbf{V}}\right],
$$

where $\mathbf{L}$ is the discretized Laplacian matrix with elements computed using Eq. (10) with cotangent weight, $\mathbf{N}$ is the vertex normal matrix, $\operatorname{diag}\left(\mathbf{s}^{\prime}\right)$ is a square diagonal matrix with the elements of the vector $\mathbf{s}^{\prime}$ which is the new signal vector including all vertices, $\mu$ is the weighting factor for vertex positions. The value of $\mu$ for boundary vertices should be set larger values than non-boundary vertices in order to prevent boundary shrinkage as the method in [21]. It is set to be 0.01 for non-boundary vertices and 1.0 for boundary vertices in our experiments, which can help us obtain satisfied results even for broken meshes. Fig. 5 shows some results in our spectral geometry processing tasks, which indicates that the Hilbert spectra generated by our method can exactly characterize the signal energy variation on 3D surface for all possible signal frequency.

To illustrate the effectiveness of the geometry processing method guided by our novel Hilbert spectra, we compare it with several related state-of-art methods in [21][24][26] by enhancing the detailed feature of the Hygeia model in Fig. 6. The method in [21] directly processes 3D surfaces by amplifying the first IMF of the input signal computed by Eq. (24). However, this simple global processing method enhances other frequency components (e.g., face and neck) and results in some distortions in Fig. 6(b) because every IMF has band overlaps in their frequency distributions. In fact, meaningful local information towards more quantitative analysis from each IMF needs to be extracted by Hilbert spectral analysis. The method in [24] uses a dimension reduction strategy based on space-filling curve to transform the HHT computation of 3D surfaces into 1D HHT computation of time series. The Hilbert spectra generated by this way is not accurate due to information loss. It can be seen that some detailed features(e.g., lips) can not be enhanced in Fig. 6(c). The enhancement result of the method based on manifold harmonic [26](Fig. 6(d)) is very close to ours(Fig. 6(e)). Both of them can enhance the detailed features accurately and retain the global shape very well.

\subsection{Prominent Local Feature Extraction}

Feature extraction and analysis plays a vital role in many graphics tasks and applications, which can assist editing, parameterization, segmentation, registration, shape retrieval, shape understanding, and many more. EMD in Hilbert-Huang transform on 3D surface decomposes a shape signal (e.g., mean curvature or Gauss curvature) into a finite number of IMFs with different feature scales, which can describe the shape geometry with a multiscale solution globally [21][23]. Its local function values of IMFs simultaneously exhibit feature information at different scales. Consequently, features can be defined as local extrema (local maximum and local minimum) of each IMF. The prominent features generally appear many times in the extrema of IMFs, therefore we could get the prominent features of a model if the appearance frequency of features at all IMFs exceed a threshold which is set to be the half of the decomposition IMF number in our experiments. The entire flowchart of this method can be described by Fig. 7.

Specifically, a vertex $\mathbf{v}_{i}$ is regarded as local maximum at each IMF $f_{k}$ if $f_{k}\left(\mathbf{v}_{i}\right)$ satisfies the following condition:

$$
\left|N_{f_{k}}^{+}\left(\mathbf{v}_{i}\right)\right| \geq t\left|N_{2}(i)\right|
$$




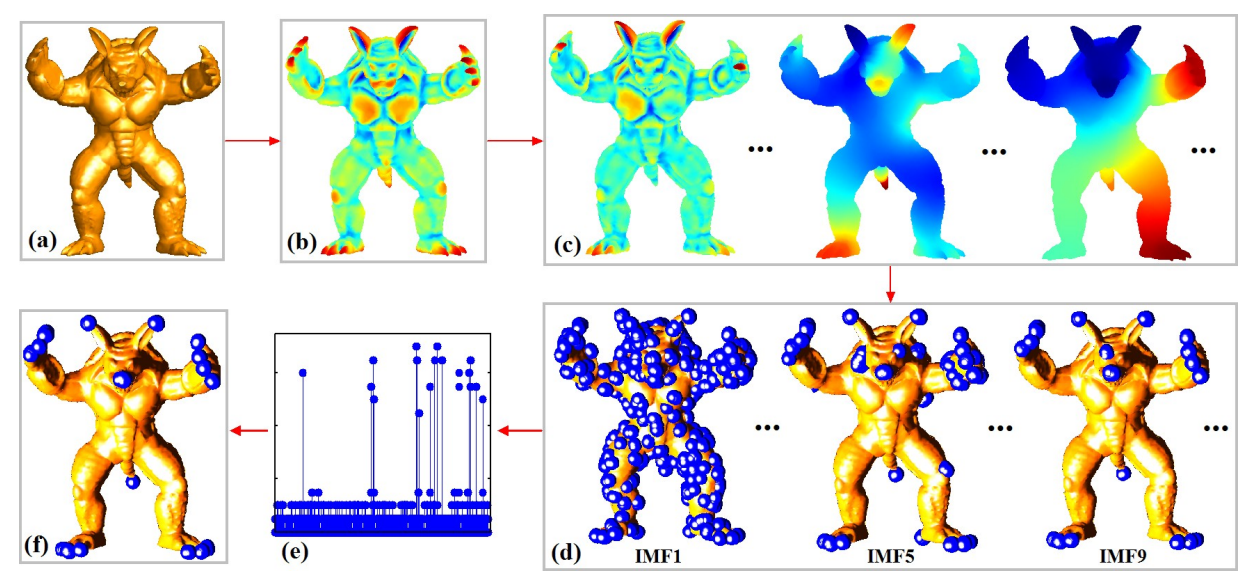

Figure 7: The flowchart of the method about prominent feature detection based on IMF extremum analysis. (a) The Armadillo model. (b) The mean curvature as an input signal. (c) IMFs generated by empirical mode decomposition. (d) The extremum points of the IMFs in (c). (e) The appearance frequency statistics of extremum points. (f) The final feature detection result.

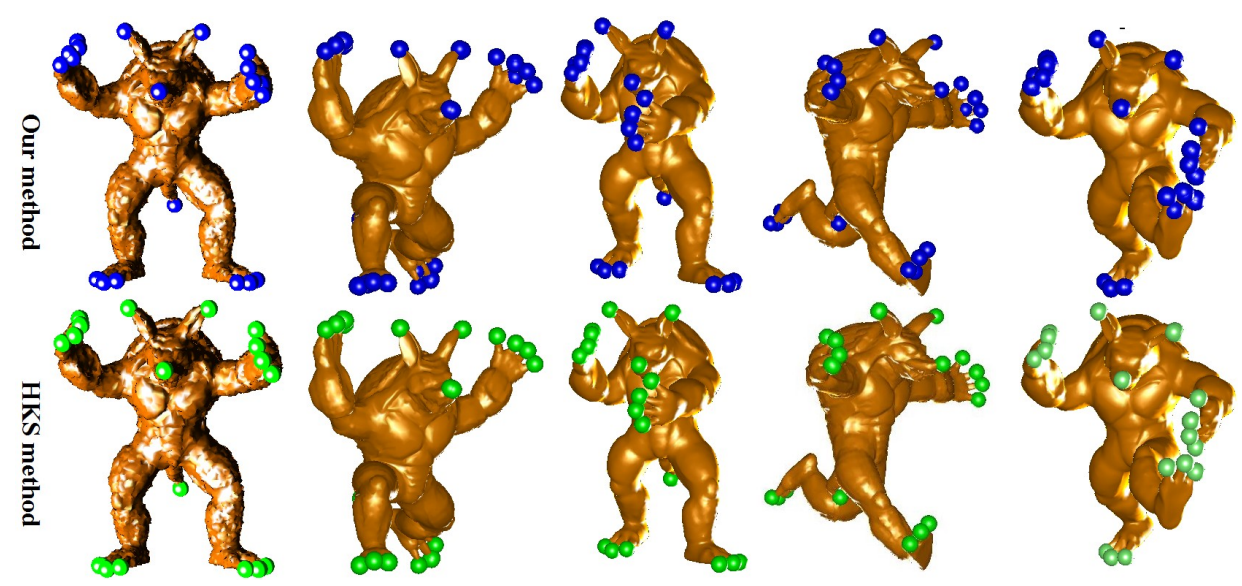

Figure 8: A comparison of prominent feature detection between our method(The first row) and HKS method [51](The second row) for the deformed Armadillo model artificially corrupted by Gaussian noise of $15 \%$ of mean edge length (left) and another four different poses of the model.

where $N_{f_{k}}^{+}\left(\mathbf{v}_{i}\right)=\left\{j \mid f_{k}\left(\mathbf{v}_{i}\right) \geq f_{k}\left(\mathbf{v}_{j}\right), j \in N_{2}(i)\right\},|\cdot|$ denotes the element number of a set, $t$ is a parameter, and $N_{2}(i)$ denotes the two-ring neighbors of $\mathbf{v}_{i}$. The local minimum can be determined in a similar fashion. If $t$ is much smaller, more extrema are involved in each IMF. We use $t=0.8$ as a default and use mean curvature as the input signal in our experiments.

In Fig. 8, we compare our method with the state-of-art method in [51] for the prominent feature detection of the deformed Armadillo model. The method in [51] extracts prominent feature vertices via the local maxima of HKS. It can be seen that these two methods produce comparable results. Both of them can extract stable prominent convex features across the different poses, and are also robust to noise corruption. Since our method incorporates the statistics of the local minimum in all IMFs, it can also extract some prominent concave features(e.g. in hands and feet) while HKS method fails to extract concave features. Besides, our method can also find other types of features by setting the input signals as different characteristics, such as texture, mass, density, conductivity, etc., as long as they are readily available. Fig. 9 illustrates more results about prominent feature detection of our method.

\subsection{Limitation and Discussion}

There are some limitations in our work which deserve discussion. First, the proposed computational scheme of Hilbert spectral analysis on 3D surfaces is much slower than its $1 \mathrm{D}$ or $2 \mathrm{D}$ counterpart which has fast algorithm to 


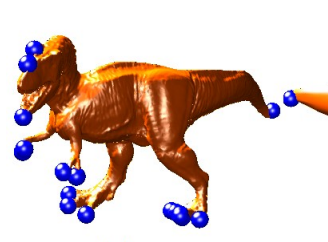

(a)

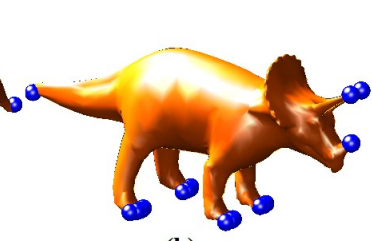

(b)

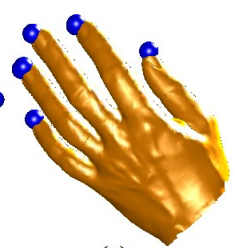

(c)

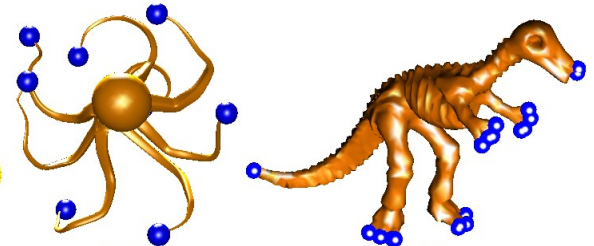

(d) (e)

Figure 9: More results about prominent feature detection. (a) Tyrannosaur model. (b) Triceratops model. (c) Hand model. (d) Octopus model. (e) Dinosaur model.

compute Hilbert transform(e.g. FFT), while Riesz transform on 3D surfaces in our scheme depends on eigenvalue decomposition of Laplacian matrix and can be costly for large data set. To ameliorate, we will investigate whether one could obtain sufficient amount of information simply by local approximation of the Laplacian operator. An alternative approach is to improve the efficiency of eigenvalue decomposition of Laplacian matrix based on its sparsity property. Second, mode mixing is a drawback of the original 1D EMD. In the 3D surface setting, it is also inherited from the original 1D EMD. The Riesz transform proposed in our paper could help reduce this side effect which characterizes the signal amplitude variation on 3D surfaces for all possible signal frequency in each IMF. However, more efficient decomposition ways as the 1D methods in [52][50] need to be explored to overcome this problem completely.

\section{Conclusion and Future Work}

In this paper, we have proposed a novel data-driven analysis theory on surfaces and have made our initial efforts to apply such theory to a few graphics applications towards more quantitative and accurate analysis. In particular, we have extended the popular Hilbert-Huang transform, a fully data-driven adaptive method, to handle signals defined over 3D surfaces. The technical core of our computational framework is to compute the Riesz transform on 3D surfaces by eigenvalue decomposition of Laplacian matrix, which utilizes the relationship between Riesz transform and fractional Laplacian operator. Furthermore, we integrate our Riesz transform with the numerical techniques of EMD on 3D surfaces by way of monogenic signals, in order to compute Hilbert spectra of any input signal defined over 3D surfaces. Hilbert spectra include the space-frequency-energy distribution of signals defined over 3D surfaces and can characterize key local feature information in a more quantitative and accurate manner. As a result, critical local information such as instantaneous frequency, local amplitude, and local phase, could aid many graphics applications, opening up a new way to process geometric and/or non-geometric information on surfaces.

Within our novel data-driven computational framework enabled by HHT on surfaces, potential applications are abundant. Through comprehensive experiments, we are able to accommodate many modeling, analysis, processing functionalities, such as 3D surface spectral processing and prominent feature detection. Extensive comparisons on popularly-used geometric models have effectively demonstrated that our surface processing method based on novel Hilbert-Huang transform on 3D surfaces can produce excellent results. Continuing to broaden the HHT's application scope to contribute to more application sub-fields would require much more research endeavors in the near future. We expect more research results on this subject to be documented in the near future by us and colleagues. Ultimately, many effective, robust analysis on a large variety of scientific datasets generated from numerous digital content paradigms would enhance the overall data processing capabilities.

\section{Acknowledgements}

This work is supported in part by National Science Foundation of USA (IIS-0949467, IIS-1047715, and IIS1049448), National Natural Science Foundation of China (61190120, 61190121, 61190125, 61202261, 61370143, 61532002), China Postdoctoral Science Foundation (2014M560875), and Scientific and Technological Development Plan of Jilin Province (20130522113JH). 


\section{References}

[1] N. E. Huang, Z. Shen, S. R. Long, M. C. Wu, H. H. Shih, Q. Zheng, N. C. Yen, C. C. Tung, H. H. Liu, The empirical mode decomposition and the Hilbert spectrum for nonlinear and non-stationary time series analysis, Proceedings of the Royal Society of London. Series A: Mathematical, Physical and Engineering Sciences 454 (1971) (1998) 903-995.

[2] N. E. Huang, Z. Wu, A review on Hilbert-Huang transform: Method and its applications to geophysical studies, Reviews of Geophysics 46 (2) (2008) 1-23.

[3] B. M. Battista, C. Knapp, T. McGee, V. Goebel, Application of the empirical mode decomposition and Hilbert-Huang transform to seismic reflection data, Geophysics 72 (2) (2007) H29-H37.

[4] J. K. Lundquist, Intermittent and elliptical inertial oscillations in the atmospheric boundary layer, Journal of the Atmospheric Sciences 60 (21) (2003) 2661-2673.

[5] C. Di, X. Yang, X. Wang, A four-stage hybrid model for hydrological time series forecasting, PLoS ONE 9 (8) (2014) e104663.

[6] J. R. Gemmrich, D. M. Farmer, Near-surface turbulence in the presence of breaking waves, Journal of Physical Oceanography 34 (5) (2004) 1067-1086

[7] W.-L. Chiang, D.-J. Chiou, C.-W. Chen, J.-P. Tang, W.-K. Hsu, T.-Y. Liu, Detecting the sensitivity of structural damage based on the HilbertHuang transform approach, Engineering Computations 27 (7) (2010) 799-818.

[8] G. Ge, L. Yu, Extrema points coding based on empirical mode decomposition: an improved image sub-band coding method, Computers \& Electrical Engineering 39 (3) (2013) 882-892.

[9] A. Linderhed, 2-D empirical mode decompositions in the spirit of image compression, in: Wavelet and Independent Component Analysis Applications IX, 1-8, 2002.

[10] Y. Tian, K. Zhao, Y. Xu, F. Peng, An image compression method based on the multi-resolution characteristics of BEMD, Computers \& Mathematics with Applications 61 (8) (2011) 2142-2147.

[11] J. C. Nunes, Y. Bouaoune, E. Delechelle, O. Niang, P. Bunel, Image analysis by bidimensional empirical mode decomposition, Image and Vision Computing 21 (12) (2003) 1019-1026.

[12] G. Xu, X. Wang, X. Xu, Improved bi-dimensional EMD and Hilbert spectrum for the analysis of textures, Pattern Recognition 42 (5) (2009) $718-734$.

[13] M.-H. Yeh, The complex bidimensional empirical mode decomposition, Signal Processing 92 (2) (2012) $523-541$.

[14] H. Ali, M. Hariharan, S. Yaacob, A. H. Adom, Facial emotion recognition using empirical mode decomposition, Expert Systems with Applications 42 (3) (2015) 1261-1277.

[15] S. Krinidis, M. Krinidis, Empirical mode decomposition on skeletonization pruning, Image and Vision Computing 31 (8) (2013) $533-541$.

[16] Y. Gao, C.-F. Li, B. Ren, S.-M. Hu, View-dependent multiscale fluid simulation, IEEE Transactions on Visualization and Computer Graphics 19 (2) (2013) 178-188.

[17] B. Ren, C.-F. Li, M. C. Lin, T. Kim, S.-M. Hu, Flow field modulation, IEEE Transactions on Visualization and Computer Graphics 19 (10) (2013) 1708-1719.

[18] D. Gabor, Theory of Communication, Journal of IEE(London) 93 (26) (1946) 429-457.

[19] S. L. Hahn, Hilbert transforms in signal processing, Artech House, 1996.

[20] D. P. Mandic, N. U. Rehman, Z. Wu, N. E. Huang, Empirical mode decomposition-based time-frequency analysis of multivariate signals: the power of adaptive data analysis, IEEE Signal Processing Magazine 30 (6) (2013) 74-86.

[21] J. Hu, X. Wang, H. Qin, Improved, feature-centric EMD for 3D surface modeling and processing, Graphical Models 76 (5) (2014) 340-354.

[22] X. Qin, X. Chen, S. Zhang, W. Wang, EMD based fairing algorithm for mesh surface, in: 11th IEEE International Conference on Computer Aided Design and Computer Graphics, 606-609, 2009.

[23] H. Wang, Z. Su, J. Cao, Y. Wang, H. Zhang, Empirical mode decomposition on surfaces, Graphical Models 74 (4) (2012) $173-183$.

[24] X. Wang, J. Hu, D. Zhang, H. Qin, Efficient EMD and Hilbert spectra computation for 3D geometry processing and analysis via space-filling curve, The Visal Computer 31 (6) (2015) 1135-1145.

[25] G. Taubin, A signal processing approach to fair surface design, in: SIGGRAPH, 351-358, 1995.

[26] B. Vallet, B. Levy, Spectral geometry processing with manifold harmonics, Comput. Graph. Forum 27 (2) (2008) $251-260$.

[27] M. Mousa, R. Chaine, S. Akkouche, E. Galin, Efficient spherical harmonics representation of 3D objects, in: Pacific Conference on Computer Graphics and Applications, 248-255, 2007.

[28] K. Zhou, H. Bao, J. Shi, 3D surface filtering using spherical harmonics, Computer-Aided Design 36 (4) (2004) $363-375$.

[29] M. Pauly, M. H. Gross, Spectral processing of point-sampled geometry, in: SIGGRAPH, 379-386, 2001.

[30] H. Zhang, O. Van Kaick, R. Dyer, Spectral mesh processing, Computer Graphics Forum 29 (6) (2010) 1865-1894.

[31] I. Guskov, W. Sweldens, P. Schröder, Multiresolution Signal Processing for Meshes, in: SIGGRAPH, 325-334, 1999.

[32] P. Schröder, W. Sweldens, Spherical wavelets: efficiently representing functions on the sphere, in: Proceedings of the 22nd Annual Conference on Computer Graphics and Interactive Techniques, SIGGRAPH '95, New York, NY, USA, ISBN 0-89791-701-4, 161-172, 1995.

[33] R. R. Coifman, M. Maggioni, Diffusion wavelets, Applied and Computational Harmonic Analysis 21 (1) (2006) 53 - 94.

[34] D. K. Hammond, P. Vandergheynst, R. Gribonval, Wavelets on graphs via spectral graph theory, Applied and Computational Harmonic Analysis 30 (2) (2011) 129-150.

[35] T. Hou, H. Qin, Admissible diffusion wavelets and their applications in space-frequency processing, IEEE Transactions on Visualization and Computer Graphics 19 (1) (2013) 3-15.

[36] T. Hou, H. Qin, Continuous and discrete Mexican hat wavelet transforms on manifolds, Graphical Models 74 (4) (2012) 221 - 232.

[37] W. H. Kim, V. Singh, M. K. Chung, C. Hinrichs, D. Pachauri, O. C. Okonkwo, S. C. Johnson, Multi-resolutional shape features via nonEuclidean wavelets: Applications to statistical analysis of cortical thickness, NeuroImage 93 (2014) 107-123.

[38] N. Li, S. Wang, M. Zhong, Z. Su, H. Qin, Generalized local-to-global shape feature detection based on graph wavelets, IEEE Transactions on Visualization and Computer Graphics .

[39] C. Li, A. B. Hamza, A multiresolution descriptor for deformable 3D shape retrieval, The Visual Computer 29 (6-8) (2013) 513-524. 
[40] M. Zhong, H. Qin, Sparse approximation of 3D shapes via spectral graph wavelets, The Visual Computer 30 (6-8) (2014) 751-761.

[41] Y. Xu, B. Liu, J. Liu, S. Riemenschneider, Two-dimensional empirical mode decomposition by finite elements, Proceedings of the Royal Society A: Mathematical, Physical and Engineering Science 462 (2074) (2006) 3081-3096.

[42] R. Pabel, R. Koch, G. Jager, A. Kunoth, Fast empirical mode decompositions of multivariate data based on adaptive spline-wavelets and a generalization of the Hilbert-Huang-transform(HHT) to arbitrary space dimensions, Advances in Adaptive Data Analysis 2 (03) (2010) $337-358$.

[43] S. Bernstein, J.-L. Bouchot, M. Reinhardt, B. Heise, Generalized analytic signals in image processing: Comparison, theory and applications, in: Quaternion and Clifford Fourier Transforms and Wavelets, 221-246, 2013.

[44] M. Felsberg, G. Sommer, The monogenic signal, IEEE Transactions on Signal Processing 49 (12) (2001) 3136-3144.

[45] E. M. Stein, Singular integrals and differentiability properties of functions, vol. 2, Princeton university press, 1970.

[46] E. M. Stein, G. L. Weiss, Introduction to Fourier analysis on Euclidean spaces, vol. 1, Princeton university press, 1971.

[47] M. Meyer, M. Desbrun, P. Schröder, A. H. Barr, Discrete differential-geometry operators for triangulated 2-manifolds, in: Visualization and Mathematics III, Mathematics and Visualization, 113-134, 2002.

[48] R. Servadei, E. Valdinoci, On the spectrum of two different fractional operators, Proceedings of the Royal Society of Edinburgh: Section A Mathematics 144 (04) (2014) 831-855.

[49] T. H. Meyer, M. Eriksson, R. C. Maggio, Gradient estimation from irregularly spaced data sets, Mathematical Geology 33 (6) (2001) 693-717.

[50] Z. Wu, N. E. Huang, Ensemble empirical mode decomposition: a noise-assisted data analysis method, Advances in Adaptive Data Analysis $1(01)(2009) 1-41$

[51] J. Sun, M. Ovsjanikov, L. Guibas, A concise and provably informative multi-Scale signature based on heat diffusion, Computer Graphics Forum 28 (5) (2009) 1383-1392.

[52] N. Pustelnik, P. Borgnat, P. Flandrin, Empirical mode decomposition revisited by multicomponent non-smooth convex optimization, Signal Processing 102 (2014) 313-331. 


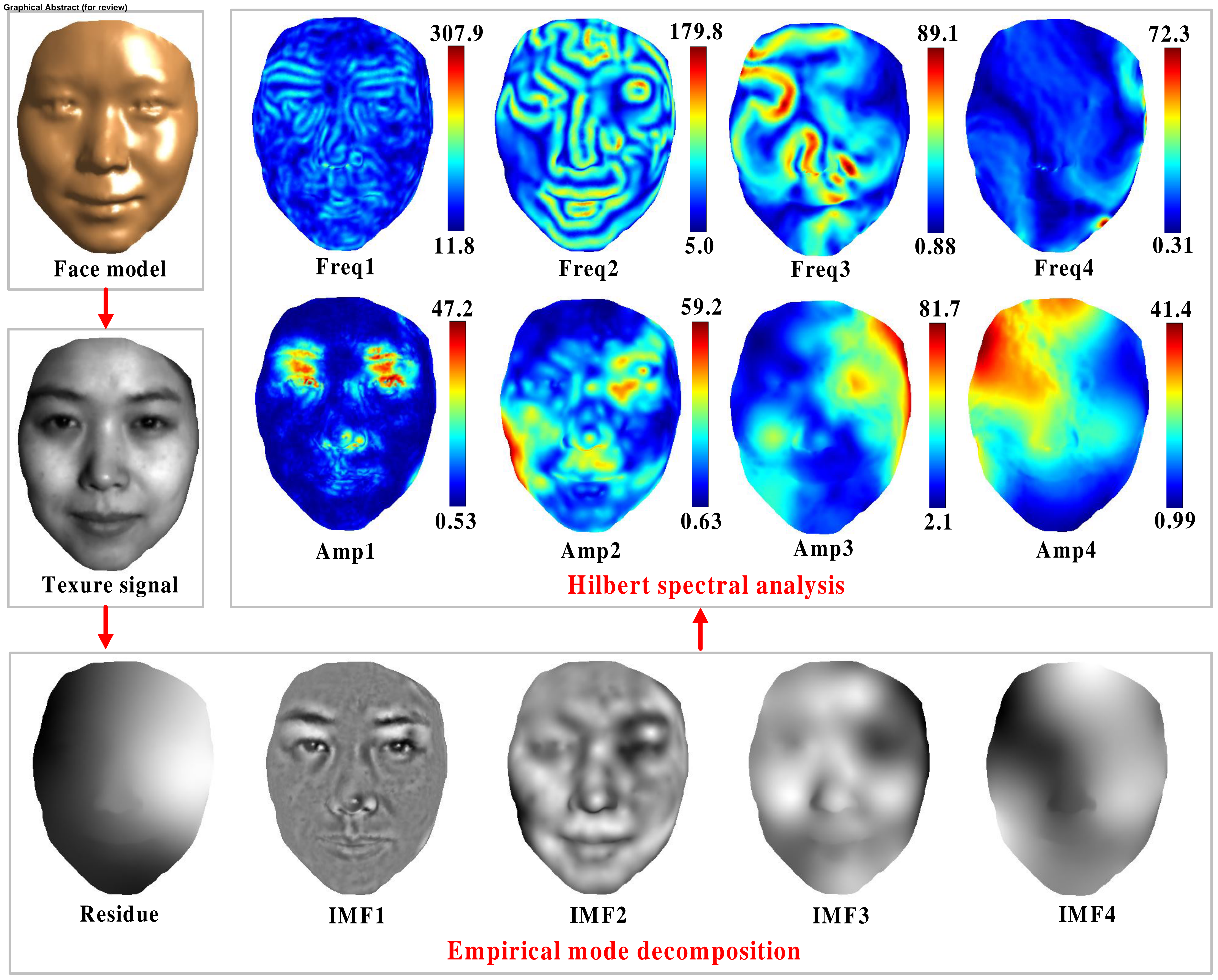

